\title{
Linking household surveys and health facility assessments to estimate intervention coverage for the Lives Saved Tool (LiST)
}

\author{
Mufaro Kanyangarara* and Victoria B. Chou
}

\begin{abstract}
Background: Calls have been made for improved measurement of coverage for maternal, newborn and child health interventions. Recently, methods linking household and health facility surveys have been used to improve estimation of intervention coverage. However, linking methods rely the availability of household and health facility surveys which are temporally matched. Because nationally representative health facility assessments are not yet routinely conducted in many low and middle income countries, estimates of intervention coverage based on linking methods can be produced for only a subset of countries. Estimates of intervention coverage are a critical input for modelling the health impact of intervention scale-up in the Lives Saved Tool (LiST). The purpose of this study was to develop a data-driven approach to estimate coverage for a subset of antenatal care interventions modeled in LiST.
\end{abstract}

Methods: Using a five-step process, estimates of population level coverage for syphilis detection and treatment, case management of diabetes, malaria infection, hypertensive disorders, and pre-eclampsia, were computed by linking household and health facility surveys. Based on data characterizing antenatal care and estimates of coverage derived from the linking approach, predictive models for intervention coverage were developed. Updated estimates of coverage based on the predictive models were compared, first with current default proxies, then with estimates based on the linking approach. Model fit and accuracy were assessed using three measures: the coefficient of determination, Pearson's correlation coefficient, and the root mean square error (RMSE).

Results: The ability to predict intervention coverage was fairly accurate across all interventions considered. Predictive models accounted for 20-63\% of the variance in intervention coverages, and correlation coefficients ranged from 0.5 to 0.83 . The predictive model used to estimate coverage of management of pre-eclampsia performed relatively better (RMSE $=0.11$ ) than the model estimating coverage of diabetes case management (RMSE $=0.19)$.

Conclusions: The new approach to estimate coverage represents an improvement over current default proxies in LiST. As the availability of reliable coverage data improves, impact estimates generated by LiST will improve. This study underscores the need for continued efforts to improve coverage measurement, while bringing to the fore the importance of health facility assessments as complementary data sources.

Keywords: Service provision assessment (SPA), Service availability and readiness assessment (SARA), Antenatal care (ANC), Lives saved tool (LiST)

\footnotetext{
* Correspondence: mkanyan1@jhu.edu

Department of International Health, Johns Hopkins Bloomberg School of

Public Health, 615 N. Wolfe Street, Baltimore, MD 21205, USA
}

(c) The Author(s). 2017 Open Access This article is distributed under the terms of the Creative Commons Attribution 4.0 International License (http://creativecommons.org/licenses/by/4.0/, which permits unrestricted use, distribution, and reproduction in any medium, provided you give appropriate credit to the original author(s) and the source, provide a link to the Creative Commons license, and indicate if changes were made. The Creative Commons Public Domain Dedication waiver (http://creativecommons.org/publicdomain/zero/1.0/) applies to the data made available in this article, unless otherwise stated. 


\section{Background}

Estimating the impact of scaling up the coverage of maternal, neonatal and child health interventions is important in evaluating progress towards national and global health goals, strategic program planning, and supporting advocacy at local, national, and global levels. The Lives Saved Tool $($ LiST) is a linear deterministic model used to estimate the impact of changes in coverage of key interventions on cause-specific maternal, neonatal, and child mortality in low and middle-income countries [1]. To model the health impact of intervention scale-up in LiST, three primary inputs are required: estimates of intervention effectiveness, measures of health and mortality status, and estimates of baseline intervention coverage [2]. Default values for these inputs are provided with the LiST software package. The flexible interface allows users to adjust or replace existing values with more recent or appropriate data. For most of the $70+$ interventions currently modeled in LiST, standard default values for coverage, formally defined as "the proportion of women and children in need of interventions who actually receive them" [3] are derived from large-scale, nationally representative household surveys such as the Demographic and Health Surveys (DHS) [4] and Multiple Indicator Cluster Surveys (MICS) [5].

Standard indicator definitions, sampling designs, and data collection tools are used in the DHS and MICS, to facilitate the tracking of intervention coverage within and between countries, and over time [6]. For example, the DHS and MICS collect data that has been used to track household ownership of insecticide-treated nets (ITNs). However, the utility of large scale household surveys in generating accurate estimates for more complex facility based interventions is limited by respondent recall, and concerns about the validity of self-reported receipt of interventions [6-8]. For example, a woman may be less certain about whether she was screened for syphilis during an ANC visit, but she may more accurately recall having her blood drawn. The validity and reliability of self-reported data represents an ongoing challenge for estimating coverage of interventions along the continuum of care [7-10].

In the absence of reliable and routinely collected data to estimate coverage, default proxies based on expert opinion and panel recommendations have been incorporated into the LiST model for some interventions. Coverage of syphilis detection and treatment during $\mathrm{ANC}$, for example, is assumed to vary based on the proportion of women who attended four or more antenatal visits $(\mathrm{ANC4}+)$ (Table 1). For case management of conditions such as diabetes, hypertensive disorders, malaria, and pre-eclampsia during pregnancy, default proxies are calculated assuming that $5 \%$ of those who attended four or more ANC visits are appropriately screened and managed (Table 1). Although this assumption recognizes that some pregnant women accessing ANC are receiving appropriate and timely case management, the designated cutoff of $5 \%$ is a rather arbitrary threshold to apply to all low and middle income countries.

For LiST, the accuracy of estimates of intervention coverage at baseline is important as misspecification may bias or skew results (i.e. inaccurate estimation of number of lives saved). As LiST is a linear model, the inaccuracy (overestimation or underestimation) of baseline coverage values will not change impact model outputs for scenarios modeling the scale-up of intervention coverage relative to the baseline (e.g. the scale up of newborn outreach interventions by $20 \%$ [11]. However,

Table 1 Definitions and current default proxy values of coverage indicators for selected interventions in the Lives Saved Tool (LiST)

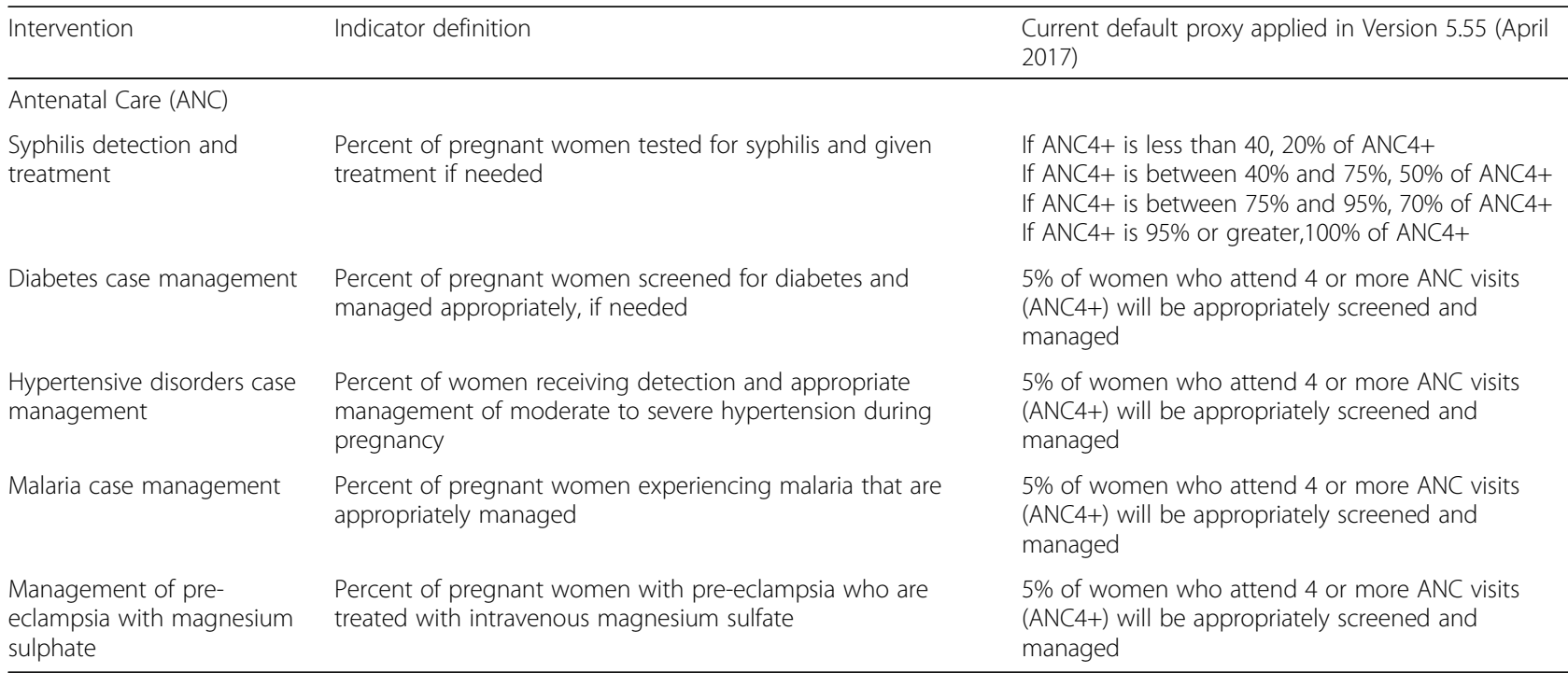


scenarios projecting toward an absolute target (e.g. an increase up to $90 \%$ as the target as used in [12] would produce impact estimates which are biased upwards, if proxy estimates for coverage are overly conservative. Conversely, if proxy estimates for coverage are overestimates of true levels of coverage, then the impact of intervention scale up determined by LiST would be inadvertently minimized.

Reliable and accurate estimates of intervention coverage are critical inputs to the LiST model, and as the availability, validity and reliability of coverage data are strengthened, impact estimates generated by LiST will improve. Coverage data may be available in some low and middle income countries, but concerns about the data quality, timeliness and reliability still persist. As part of the broader agenda to end preventable maternal, neonatal and child deaths, calls have been made for improved coverage measurement to track population coverage for life-saving maternal, newborn and child health interventions $[3,13]$. Technical work is ongoing to harmonize survey tools and increase the validity and reliability of a core set of indicators used for global monitoring of intervention coverage $[6,9,14]$. One promising approach to improve coverage measurement relies on linking self-reported care-seeking data collected through household surveys to data on service availability and readiness from health facility assessments. There is increasing recognition that this strategy- hereafter referred to as the 'linking approach' may be a feasible option for estimating coverage of interventions not amenable to tracking by household surveys alone $[15,16]$.

Although the linking of household and health facility surveys represents improved estimates of intervention coverage when no routine coverage data exists, the linking approach depends on the availability and temporal alignment of household and health facility surveys. Although many health facility assessment tools have been developed, nationally representative health facility assessments are not yet routinely conducted and survey data available in many low and middle income countries [17]. For the purposes of LiST modeling, it remains crucial to estimate intervention coverage for all low and middle income countries using the available data to inform the process. The objective of this study was to use estimates of intervention coverage derived from the linking approach to guide the development of formulas to calculate new estimates for intervention coverage in LiST. For a subset of ANC interventions, we estimated population-level coverage based on the linking approach, then compared these to the existing proxies in the most recent version of the LiST model (Spectrum version 5.55, released April 14, 2017). By applying a simple predictive modeling framework, we developed updated estimates for coverage of syphilis detection and treatment, case management of diabetes, hypertensive disorders, malaria infection, and pre-eclampsia. Lastly, we provided recommendations to guide the inclusion of improved estimates of coverage of maternal, newborn and child health interventions in LiST. This study underscores the need for continued efforts to improve coverage measurement, and highlights the importance of health facility assessments as valuable data sources.

\section{Methods}

We used a five step process to develop updated estimates of baseline intervention coverage for syphilis detection and treatment, case management of diabetes, hypertensive disorders, malaria infection, and preeclampsia (Fig. 1). First, we used data collected from two large-scale nationally representative health facility assessments, the Service Provision Assessment (SPA) and the Service Availability and Readiness Assessment (SARA), to calculate health facility 'readiness' to deliver each intervention. 'Readiness' was defined by the availability of the relevant drugs, equipment, supplies, guidelines and trained staff necessary to deliver a specific intervention. Facility-level indicators were summarized at the stratum level as the proportion of health facilities ready to deliver the intervention in that stratum. Strata were defined by health facility type (hospital, health center, health post, etc.), managing authority (public, nonpublic) and location (rural, urban).

Second, we estimated coverage of ANC4+ within each stratum, using data on care-seeking from the DHS. The woman's questionnaire of the DHS collects information on pregnancy-related care for the most recent live births occurring within 5 years prior to the survey. To reduce recall bias, this analysis was restricted to reports about ANC received for live births occurring only within the 3 years prior. The analysis was also restricted to the sample of women who reported attending at least four ANC visits, as most ANC interventions we considered require more than one ANC visit to be delivered at sufficient quality to have an impact on the intended health outcomes. Also, ANC4+ coverage is a standard indicator used in LiST. Based on the reported source of ANC (managing authority and health facility type) and residence (urban/rural), we computed the distribution of ANC4+ coverage by stratum (health facility type, managing authority and residence).

Third, we computed the product of ANC coverage and health facility readiness for each stratum, then summed across all strata. The resulting estimates represented the proportion of women who attended ANC at least four times and sought care at a health facility 'ready' to deliver the specific intervention. Of note, these estimates represent the proportion of women for whom 


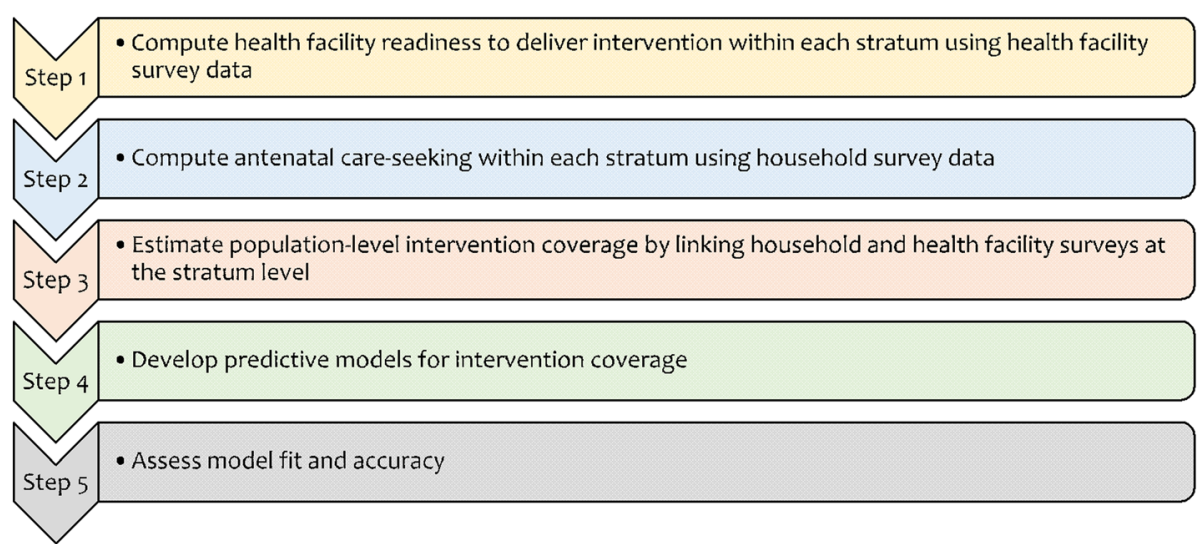

Fig. 1 Five step process to develop formulas to update estimates of intervention coverage in the Lives Saved Tool

an intervention was available (also referred to 'availability coverage') [18], but several factors related to clinical practice could hinder women from actually receiving the intervention [19]. Country-specific coverage values for each intervention were available for 20 SPAs or SARAs conducted in 13 sub-Saharan African countries with a DHS conducted 2 years prior or after the SPA/SARA. The full list of countries where coverage could be estimated based on the linking approach were Benin, Burkina Faso, Democratic Republic of Congo, Ghana, Kenya, Namibia, Rwanda, Senegal, Sierra Leone, Tanzania, Togo, Uganda, and Zimbabwe. Further details on the indicator definitions and exclusion criteria can be found in Additional files 1, 2 and 3.

Fourth, we developed simple predictive models for intervention coverage, with the goal to use these in LiST to estimate intervention coverage for all low and middle income countries. The pool of candidate independent variables was drawn from existing DHS data characterizing ANC, specifically the number of ANC visits, timing of ANC visits, and receipt of ANC components: measurement of blood pressure, height and weight, and collection of urine and blood samples. These variables were selected based on both the availability in the DHS and the plausibility of an association with intervention coverage, as they represent elements of ANC essential for the delivery of key ANC interventions. For example, measurement of blood pressure is used to screen pregnant women for hypertensive disorders.

Using the country-level estimates of coverage from the linking approach as the outcomes, we specified fractional logit models, with a logit link and binomial error distribution, to determine variables that predict coverage [20]. To identify factors predictive of intervention coverage, we conducted stepwise regression analysis with exit criteria set at the $p=0.1$ level. Given the small sample size $(n=20)$, the number of parameters in the predictive models was restricted to a maximum of two (excluding the intercept). Assessing for interaction terms, non- linear relationships, and the inclusion of more independent variables may have strengthened our analysis, but was not conducted due to concerns about the sample size.

Lastly, we considered the fit and accuracy of the predictive models using three metrics: the coefficient of determination $\left(\mathbb{R}^{2}\right)$, Pearson's correlation coefficient $(\rho)$ and the root mean square error (RMSE). A higher coefficient of determination and lower value of RMSE indicate better model fit. Correlations above 0.8 were deemed indicative of strong associations, and those in the $0.5-0.8$ range were considered moderate associations.

To assess the impact of different baseline values on estimates of the lives saved in the LiST model, we considered the scale-up of coverage of magnesium sulphate for the management of pre-eclampsia to $90 \%$, first using default proxy values, then using coverage estimates computed from the prediction model as baseline values of coverage. The year 2015 was used as the baseline year and the target year was the subsequent year. The coverage of all other interventions in LiST was held constant to ensure that only the effect of the specific intervention of interest on mortality was modelled. The number of lives saved were estimated for each of the 13 sub-Saharan Africa countries and the difference in impact produced by LiST using the different baseline values compared.

All estimates accounted for the complex survey sampling (cluster design and sampling weights) by using svyset command. All analyses were conducted using STATA (College Station, Texas) version 14.2 and current default proxies used as defaults were based on Spectrum version 5.55 (released April 14, 2017) of LiST.

\section{Results}

Comparison of current default proxies with estimates from the linking approach

For coverage of case management of diabetes, hypertensive disorders, malaria infection, and pre-eclampsia, 
comparison of current default proxy values with estimates generated by the linking approach as the "gold standard" indicated that current default proxy values are overly conservative (Fig. 2). Furthermore, the extent to which current default proxy values underestimated coverage varied by intervention, with coverage of case management of malaria infection showing the widest gap, and coverage of case management of diabetes case representing the narrowest. For coverage of syphilis detection and treatment, there was no clear tendency for the existing default proxy values to underestimate or overestimate intervention coverage as estimated by the linking approach. The differences between current default proxy values and estimates from the linking approach ranged from $-45 \%$ to $34 \%$.

\section{Predictive models for intervention coverage}

From the multivariate analyses, the rate of blood collection and rate of early ANC initiation ( $<4$ months) predicted intervention coverage for syphilis detection and treatment (Table 2). Coverage of hypertensive disorder case management was positively predicted by the rate of blood pressure measurement. The rate of blood collection was a determinant of coverage for both diabetes case management and malaria case management. A higher rate of blood collection together with a higher ANC4+ coverage predicted higher coverage of management of pre-eclampsia.

\section{Comparison of updated estimates with estimates from the linking approach}

Figure 2 shows the comparison of updated estimates of coverage based on the prediction models versus estimates from the linking approach by intervention. The dashed line represents perfect prediction. The model for coverage of hypertensive disorder case management performed reasonably well with the least scatter around the perfect prediction line. Estimates from models for coverage of syphilis detection and treatment and coverage of malaria case management exhibited more scatter around
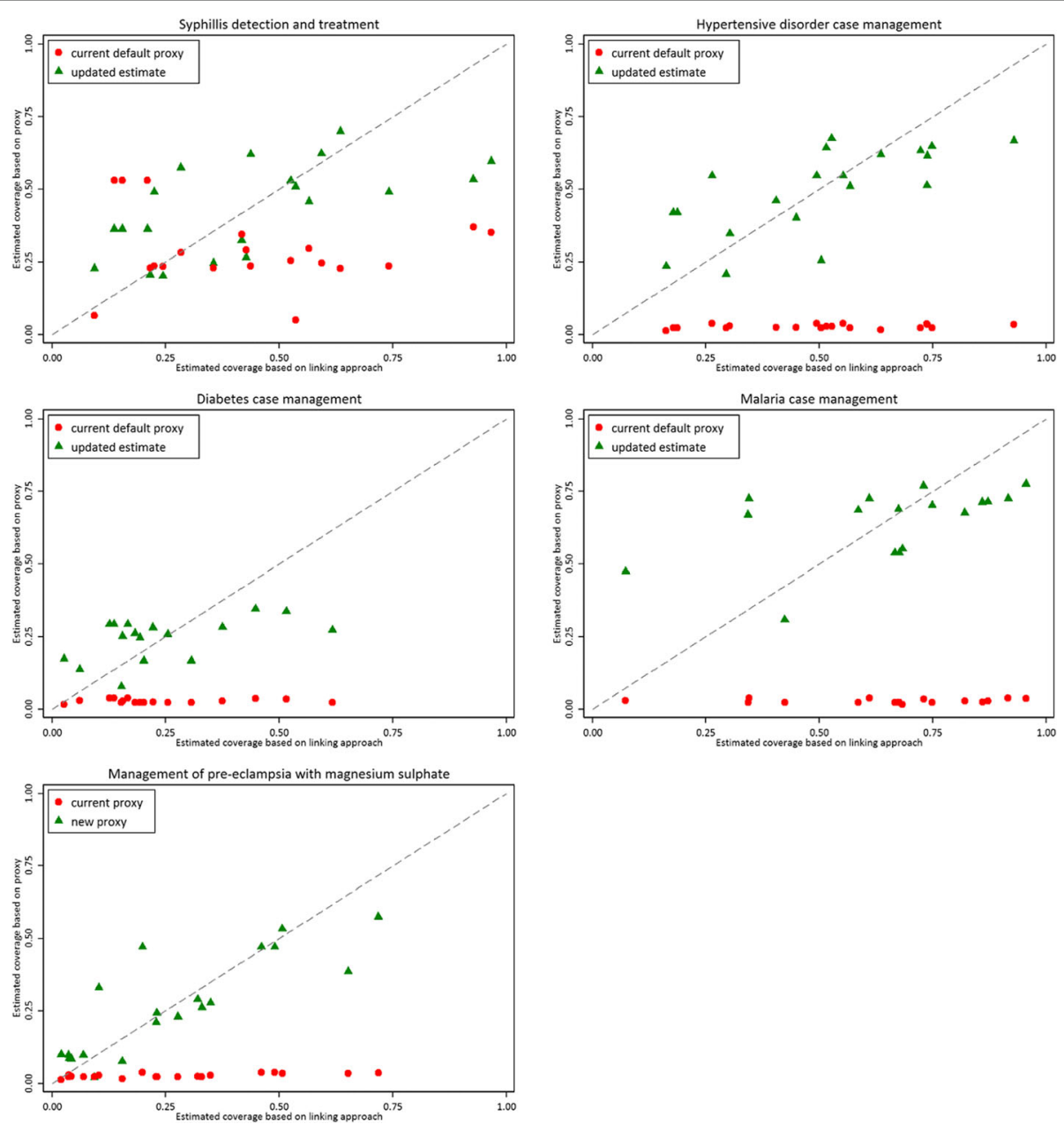

Fig. 2 Comparison of current default proxy, linking estimates, and updated estimates of coverage for selected antenatal care interventions 
Table 2 Multivariate predictors of ANC intervention coverage

\begin{tabular}{|c|c|c|c|c|c|c|c|c|c|c|}
\hline & \multicolumn{2}{|c|}{ Syphilis detection and treatment } & \multicolumn{8}{|c|}{ Case management } \\
\hline & \multirow[b]{2}{*}{ Estimate } & \multirow[b]{2}{*}{$p$} & \multicolumn{2}{|l|}{ Diabetes } & \multicolumn{2}{|c|}{ Hypertensive disorders } & \multicolumn{2}{|c|}{ Malaria infection } & \multicolumn{2}{|c|}{ Pre-eclampsia } \\
\hline & & & Estimate & $p$ & Estimate & $p$ & Estimate & $p$ & Estimate & $\mathrm{p}$ \\
\hline Intercept & -1.22 & 0.17 & -3.21 & 0.001 & -1.62 & 0.001 & -1.64 & 0.10 & -6.44 & $<0.001$ \\
\hline Blood sample taken & 3.36 & 0.02 & 2.61 & 0.03 & & & 2.92 & 0.02 & 4.91 & 0.002 \\
\hline Urine sample taken & & & & & 2.50 & $<0.001$ & & & & \\
\hline Early ANC enrolment^ & -5.23 & $<0.001$ & & & & & & & & \\
\hline Attended 4 or more ANC visits & & & & & & & & & 2.56 & 0.05 \\
\hline
\end{tabular}

$\overline{A N C}$ antenatal care, ${ }^{\wedge}$ Early ANC enrolment was defined as first ANC visit within the first 4 months of pregnancy

the perfect prediction line, with several outliers far from the perfect prediction line, and very little agreement.

The measures assessing model fit and accuracy of the five prediction models are summarized in Table 3. Correlation coefficients ranged from $0.5-0.83$ indicating moderate to strong agreement between updated estimates and coverage estimates from the linking approach. Independent variables included in predictive models explained between 20 and $63 \%$ of the variability in intervention coverage. Overall, the predictive model for case management for pre-eclampsia had relatively good accuracy $(\mathrm{RMSE}=0.11)$. By contrast, the predictive model for coverage of diabetes case management had a poorer model fit $($ RMSE $=0.19)$.

\section{Comparison of model outputs using updated estimates and current default proxies as baseline coverage}

We assessed difference in impact estimates for the scaleup of coverage of magnesium sulphate for the management of pre-eclampsia to $90 \%$ applying the two difference baseline coverage estimates. Given the universally lower default proxy values of coverage, scale up of coverage to $90 \%$ in the following year resulted in marginally diminished impact estimates using the updated estimates than default proxy values (Table 4). For Burkina Faso where there was a negligible difference in baseline values (current default: $2 \%$ vs updated: $8 \%$ ), there were similarly negligible differences in maternal lives saved (3.7\%) and stillbirths prevented (2.2\%). By contrast, for Ghana which represented the widest gap in baseline values (current default: $4 \%$ vs linking: 60\%), impact estimates dropped to $47 \%(84 / 177)$ and $60 \%(532 / 885)$ of the original maternal and stillbirth impact respectively.

\section{Discussion}

We developed formulas to calculate updated estimates for the coverage of syphilis detection and treatment, case management of diabetes, malaria infection, hypertensive disorders, and pre-eclampsia. The updated estimates of coverage offer a few important advantages over the current default proxies in LiST. Our approach to coverage estimation was data-driven, making use of countryspecific information collected through household and health facility surveys. Health facility assessments represent a rich, yet under-utilized source of information about the performance of health systems. To date, over 48 SPAs and SARAs have been conducted globally, with the majority in sub-Saharan Africa (Additional file 2). Other health facility assessments have been developed, including the Facility Audit of Service Quality (FASQ), Health Facility Census (HFC), and Service Availability Mapping (SAM). As more health facility assessments are conducted and implementation becomes more routine, the geographic coverage and generalizability of findings from health facility assessments will expand. Recently, three SPAs were been implemented outside of subSaharan Africa, in Bangladesh (2014), Haiti (2013), and Nepal (2015).

Updated estimates for coverage accounted for the heterogeneity in coverage by intervention not evident in current default proxies, and indicated that current default proxies are most likely conservative. For example, current default proxies for coverage of case management of diabetes, hypertensive disorders, malaria infection, and pre-eclampsia during pregnancy are based on the blanket assumption of that coverage equals $5 \%$ of ANC4 + coverage. While the current default proxies are likely

Table 3 Model performance statistics for models predicting intervention coverage

\begin{tabular}{llll}
\hline & R squared & Correlation & Root mean square error \\
\hline Syphilis detection and treatment & 0.38 & $0.63(p=0.002)$ & 0.19 \\
Diabetes case management & 0.20 & $0.50(p=0.04)$ & 0.14 \\
Hypertensive disorder case management & 0.47 & $0.70(p<0.001)$ & 0.15 \\
Malaria case management & 0.28 & $0.52(p=0.03)$ & 0.19 \\
Management of pre-eclampsia with magnesium sulphate & 0.63 & $0.83(p<0.001)$ & 0.11 \\
\hline
\end{tabular}


Table 4 Comparison of impact estimates for the scale up of coverage of magnesium sulphate for management of pre-eclampsia to $90 \%$

\begin{tabular}{|c|c|c|c|c|c|c|c|}
\hline & \multirow{2}{*}{\multicolumn{3}{|c|}{ Baseline coverage (\%) }} & \multicolumn{4}{|c|}{ Impact estimates } \\
\hline & & & & \multicolumn{2}{|c|}{ Maternal lives saved } & \multicolumn{2}{|c|}{ Stillbirths prevented } \\
\hline & Current & Updated & Diff & Current & Updated & Current & Updated \\
\hline Benin & 3 & 28 & 25 & 117 & 98 & 497 & 445 \\
\hline Burkina Faso & 2 & 8 & 6 & 273 & 263 & 435 & 425 \\
\hline DRC & 2 & 10 & 7 & 2338 & 2236 & 2070 & 2015 \\
\hline Ghana & 4 & 64 & 60 & 177 & 84 & 885 & 532 \\
\hline Kenya & 3 & 43 & 40 & 804 & 571 & 87 & 70 \\
\hline Namibia & 3 & 50 & 47 & 17 & 11 & 34 & 26 \\
\hline Rwanda & 2 & 37 & 34 & 124 & 95 & 295 & 249 \\
\hline Senegal & 2 & 26 & 24 & 186 & 158 & 281 & 254 \\
\hline Sierra Leone & 4 & 47 & 43 & 313 & 211 & 278 & 215 \\
\hline Tanzania & 2 & 29 & 27 & 717 & 594 & 641 & 570 \\
\hline Togo & 3 & 33 & 30 & 88 & 71 & 380 & 330 \\
\hline Uganda & 2 & 24 & 22 & 623 & 539 & 1638 & 1497 \\
\hline Zimbabwe & 4 & 58 & 54 & 153 & 84 & 486 & 326 \\
\hline
\end{tabular}

Current $=$ current default proxy value in LiST; Updated = updated estimates based on formulas; Diff = absolute difference between current default proxy and updated estimate

an underestimation of true intervention coverage, the resulting bias may not be as apparent when examining mortality reduction as a result of a relative increase in coverage. However, the accuracy of baseline coverage becomes more critical when LiST users are interested in projecting the impact of an absolute or universal scaleup. While current default proxies of coverage have been useful in establishing baseline coverage where no routine coverage data exists, as strategies for coverage measurement improve, it is important to continuously update estimates of intervention coverage in LiST. More reliable estimates of coverage will inform the setting more appropriate and actionable target goals for coverage, and improve the estimation of projected impact of scaling up intervention coverage in LiST.

Our development of updated estimates is based on estimates of coverage derived from linking household and health facility surveys, a methodology that has not been validated, and presents several challenges. Notably, the linking approach relies on the availability of temporally aligned household and health facility surveys. Despite the fairly inclusive definition of temporal alignment $(+/-$ 2 years) used in the present study, our sample size was small, with only 20 health facility surveys from 13 countries in sub-Saharan Africa. As a result, the updated estimates determined in the current study may not truly represent coverage of these interventions in all low and middle income countries. The small sample size also restricted the statistical methods and inclusion of independent variables. As more data become available and constraints due to a small sample size are eased, the inclusion of a broader range of independent variables and use of more sophisticated statistical methods may produce different models and potentially more reliable estimates of country-specific coverage.

Another limitation is related to the assumptions underlying the linking method used. We derived estimates of coverage based on the linking approach, which theoretically determined the proportion of women attending ANC at a health facility deemed 'ready' to provide a specific intervention. Readiness was categorized by specifying the minimum conditions necessary to deliver a particular intervention. For example, a health facility was judged to be ready to deliver syphilis detection and treatment if at least one valid syphilis test and drug to treat syphilis were observed in the health facility on the day of assessment. The definitions of health facility readiness for each intervention affected the estimates of coverage obtained by the linking approach. As linking was done at the stratum, and not individual level, large variations in health facility readiness between facilities in the same stratum (health facility type, managing authority and location) will affect the reliability of estimates. Furthermore, factors such as health worker knowledge, supervision and work load will likely reduce the overall proportion of women in need of an intervention who received it [19]. However, it is not possible to determine the extent to which these factors led to biased estimates of coverage.

Ideally, low and middle income countries should strive to establish robust routine health information systems. 
Unfortunately, given the current challenges it will be necessary to rely on linking household and health facility surveys for the foreseeable future $[3,16,21]$. The combination of two surveys - household surveys to assess care-seeking and health facility assessments to assess the availability and readiness of the health system - will provide more reliable data to track progress towards ending preventable maternal, neonatal and child deaths. The linking approach is a promising strategy, but further research to develop and validate the methods is warranted. Such studies should assess the accuracy and validate estimates of coverage derived from the linking approach. It is important to determine whether linking methods at aggregate levels are sufficiently reliable to determine level of intervention coverage quantitatively and thus, able to be used to track progress over time. Further standardization of health facility assessments and standard definitions of readiness are needed.

\section{Conclusions}

In summary, we updated estimates of coverage for syphilis detection and treatment, case management of diabetes, hypertensive disorders, malaria infection, and preeclampsia for use in LiST. Work is ongoing to use linking methods to improve estimation of coverage of other interventions along the continuum, where LiST currently uses proxy assumptions. While more work is needed to improve coverage measurement for maternal, neonatal and child health interventions more generally, our updated estimates facilitate the establishment of baseline coverage in LiST. By balancing the desire for robust estimates of intervention coverage with the limitations of sparse data availability in low and middle income countries, we accomplished the task of improving proxy estimates of intervention coverage, a critical input in LiST.

\section{Additional files}

Additional file 1: Table: Indicator definitions. (DOCX $17 \mathrm{~kb}$ )

Additional file 2: Table: List of Service Provision Assessments (SPA) and Service Availability and Readiness Assessments (SARA) identified. (DOCX 19 kb)

Additional file 3: Figure: Flow chart of exclusion criteria. (DOCX 28 kb)

\section{Abbreviations}

ANC: Antenatal care; FASQ: Facility Audit of Service Quality; HFC: Health Facility Census; LiST: Lives Saved Tool; RMSE: Root mean square error; SAM: Service Availability Mapping; SARA: Service Availability and Readiness Assessment; SPA: Service provision assessment

\section{Acknowledgements}

We would like to thank Neff Walker for his valuable contribution and input about the study design, interpretation, and presentation of the findings.

\section{Funding}

The publication costs for all supplement articles were funded by a grant from the Bill \& Melinda Gates Foundation (JHU Grant 115,621, Award Number OPP1084423 for the "Development and Use of the Lives Saved Tool (LiST)").

\section{Availability of data and materials}

N/A.

\section{About this supplement}

This article has been published as part of BMC Public Health Volume 17 Supplement 4, 2017: The Lives Saved Tool in 2017: Updates, Applications, and Future Directions. The full contents of the supplement are available online at https://bmcpublichealth.biomedcentral.com/articles/supplements/ volume-17-supplement-4.

\section{Authors' contributions}

MK and VBC jointly conceived the paper and MK conducted all the analyses. MK drafted the manuscript. All authors read and approved the final manuscript.

\section{Competing interest}

The authors declare that they have no competing interests.

\section{Ethics approval and consent to participate}

N/A.

\section{Consent for publication}

N/A.

\section{Publisher's Note}

Springer Nature remains neutral with regard to jurisdictional claims in published maps and institutional affiliations.

Published: 7 November 2017

\section{References}

1. Walker N, Tam Y, Friberg IK. Overview of the lives saved tool (LiST). BMC Public Health. 2013;13(Suppl 3):S1.

2. Winfrey W, McKinnon R, Stover J. Methods used in the Lives Saved Tool (LiST). BMC Public Health. 2011;11(3):S32.

3. Bryce J, Arnold F, Blanc A, Hancioglu A, Newby H, Requejo J, et al. Measuring Coverage in MNCH: New Findings, New Strategies, and Recommendations for Action. PLoS Med. 2013;10(5):e1001423.

4. Demographic and Health Surveys [Internet]. [cited 2016 Jan 1]. Available from: http://www.dhsprogram.com

5. Statistics and Monitoring: Multiple Indicator Cluster Survey [Internet]. [cited 2016 Jan 1]. Available from: http://mics.unicef.org/

6. Hancioglu A, Arnold F. Measuring Coverage in MNCH: Tracking Progress in Health for Women and Children Using DHS and MICS Household Surveys. PLoS Med. 2013;10(5):1-8.

7. Stanton CK, Rawlins B, Drake M, dos Anjos M, Cantor D, Chongo L, et al. Measuring Coverage in MNCH: Testing the Validity of Women's Self-Report of Key Maternal and Newborn Health Interventions during the Peripartum Period in Mozambique. PLoS One. 2013;8(5):1-11.

8. Blanc AK, Warren C, McCarthy KJ, Kimani J, Ndwiga C, RamaRao S. Assessing the validity of indicators of the quality of maternal and newborn health care in Kenya. J Glob Health. 2016;6(1):10405.

9. McCarthy KJ, Blanc AK, Warren CE, Kimani J, Mdawida B, Ndwidga C. Can surveys of women accurately track indicators of maternal and newborn care? A validity and reliability study in Kenya. J Glob Health. 2016;6(2):20502.

10. Montgomery A, Goufodji S, Kanhonou L, Alihonou E, Collin S, Filippi V. Validity and Reliability of Postpartum Morbidity Questionnaires in Benin. Matern Child Health J. 2012;16(8):1728-31.

11. Friberg IK, Kinney MV, Lawn JE, Kerber KJ, Odubanjo MO, Bergh A-M, et al. SubSaharan Africa's Mothers, Newborns, and Children: How Many Lives Could Be Saved with Targeted Health Interventions? PLoS Med. 2010;7(6):1-8.

12. Marsh A, Munos M, Baya B, Sanon D, Gilroy K, Bryce J. Using LiST to model potential reduction in under-five mortality in Burkina Faso. BMC Public Health 2013 17;13(Suppl 3):S26-S26.

13. Marchant T, Bryce J, Victora C, Moran AC, Claeson M, Requejo J, et al. Improved measurement for mothers, newborns and children in the era of the Sustainable Development Goals. J Glob Health. 2016:6(1):10506.

14. Requejo $\mathrm{JH}$, Newby H, Bryce J. Measuring Coverage in $\mathrm{MNCH}$ : Challenges and Opportunities in the Selection of Coverage Indicators for Global Monitoring. PLoS Med. 2013;10(5):1-8. 
15. Munos MK, Stanton CK, Bryce J. The Core Group for Improving Coverage Measurement for $\mathrm{MNCH}$. Improving coverage measurement for reproductive, maternal, neonatal and child health: gaps and opportunities. J Glob Health. 2017;7(1):010801.

16. Do M, Micah A, Brondi L, Campbell H, Marchant T, Eisele T, et al. Linking household and facility data for better coverage measures in reproductive, maternal, newborn, and child health care: systematic review. J Glob Health. 2016 Dec 3;6(2):20501

17. Nickerson JW, Adams O, Attaran A, Hatcher-Roberts J, Tugwell P. Monitoring the ability to deliver care in low- and middle-income countries: a systematic review of health facility assessment tools. Health Policy Plan. 2015;30(5):675.

18. Tanahashi T. Health service coverage and its evaluation. Bull World Health Organ. 1978;56(2):295-303

19. Baker U, Okuga M, Waiswa P, Manzi F, Peterson S, Hanson C. Bottlenecks in the implementation of essential screening tests in antenatal care: Syphilis, HIV, and anemia testing in rural Tanzania and Uganda. Int I Gynaecol Obstet. 2015;130(Suppl):S43-50.

20. Papke $L E$, Wooldridge J. Econometric methods for fractional response variables with an application to 401 (k) plan participation rates. National Bureau of Economic Research Cambridge, Massachusetts, USA; 1993.

21. Frøen JF, Friberg IK, Lawn JE, Bhutta ZA, Pattinson RC, Allanson ER, et al. Stillbirths: progress and unfinished business. Lancet. 2016;387(10018):574-86.

\section{Submit your next manuscript to BioMed Central} and we will help you at every step:

- We accept pre-submission inquiries

- Our selector tool helps you to find the most relevant journal

- We provide round the clock customer support

- Convenient online submission

- Thorough peer review

- Inclusion in PubMed and all major indexing services

- Maximum visibility for your research

Submit your manuscript at www.biomedcentral.com/submit 